\title{
Collision tumor with inflammatory breast carcinoma and malignant phyllodes tumor: a case report and literature review
}

\author{
Young Duck Shin ${ }^{1}$, Seul Kee Lee ${ }^{2}$, Kyu Sun Kim³ ${ }^{3}$ Mi Ja Park ${ }^{4}$, Joo Heon Kim ${ }^{4}$ Hyun Sun Yim and Young Jin Choi ${ }^{2 *}$
}

\begin{abstract}
There have been some reports of coincidental presentation of breast carcinoma and phyllodes tumor in the same breast. Most of the cases were carcinoma that arose from a phyllodes tumor with a histologically identified transitional area, and they behaved less aggressively than the usually encountered carcinoma. Collision tumors are rare clinical entities in which two histologically distinct tumor types show involvement at the same site. The occurrence of these tumors in the breast is extremely rare. Here, we report a case of 45 -year-old woman who had both invasive ductal carcinoma as the finding of inflammatory carcinoma and a malignant phyllodes tumor in the same breast. There was no evidence of a transitional area between the phyllodes tumor and the invasive ductal carcinoma. To our knowledge, this is the first report of a collision tumor of inflammatory breast carcinoma coincident with a malignant phyllodes tumor in same breast.
\end{abstract}

Keywords: Breast, Neoplasm, Phyllodes tumor, Ductal carcinoma, Inflammatory breast carcinoma

\section{Background}

Collision tumors are rare clinical entities in which two histologically distinct tumor types show involvement in the same site. Coincidental occurrence of breast carcinoma and axillary lymphoma has been reported with a variant subtype of lymphoma and leukemia [1,2]. But, the presentation of carcinoma and other tumors in same breast as a finding of collision tumor is extremely rare, and there have been few reports of collision tumor consisting of invasive ductal carcinoma admixed with breast mucosa-associated lymphoid tissue lymphoma [3], chronic lymphocytic leukemia and lactating adenoma [4]. The epithelial component of phyllodes tumors (PTs) can transform to carcinoma and the type of carcinoma that has been reported varied and include in situ carcinoma invasive ductal, tubular, lobular and squamous carcinoma types [5-7]. These lesions reported in the literature showed a histologically identified transitional area, and they behaved less aggressively than the usually encountered carcinoma. The present case of collision tumor, which consisted of PT and

\footnotetext{
* Correspondence: yj0139@naver.com

${ }^{2}$ Department of Surgery, Eulji University Hospital, Eulji University School of Medicine, Dunsan-dong 1306, Seo-gu, Daejeon 135-710, Korea

Full list of author information is available at the end of the article
}

invasive carcinoma in the same breast, is extremely rare;in a review of the literature, we found three cases of collision tumor with malignant PT and invasive carcinoma [8-10].

Here, we report a case of a 45-year-old woman who had both malignant PT with invasive carcinoma as the finding of inflammatory breast carcinoma with massive lymph node (LN) involvement. There was no evidence of a definite transition between two types of tumor, the patient was diagnosed as having a collision tumor with carcinoma and malignant PT. To our knowledge, this is the first report of a collision tumor of the breast that consisted of inflammatory carcinoma and malignant PT.

\section{Case presentation}

A 45-year old woman presented with a huge left breast mass that had necrosis and a foul odor. She had a history of contralateral metaplastic carcinoma, and she had undergone a right modified radical mastectomy 15 years previously. She also presented with a 2-cm-sized painless palpable breast mass in her left breast two years before presentation for the case in this report, but she did not undergo any clinical work-up at that time. Then, approximately 2 or 3 months prior to presentation of this case, she noticed a mass extrusion of the outside skin with rapid 
growth. On physical examination, a huge necrotic mass $20 \mathrm{~cm}$ in diameter was seen in the entire left breast. The mass was extruded skin outside, and skin overlying the mass was thickened with edematous change (Figure 1). The axilla showed multiple conglomerated lymph nodes (LNs) that were fixed to the chest wall.

A chest computerized tomography (CT) showed a 22$\mathrm{cm}$-sized huge breast mass with necrotic changes and multiple LN enlargements in the left axilla, both supraclavicular and parasternal areas including the left internal mammary and paracardiac LNs (Figure 2a). On ${ }^{18}$ F-FDG positron emission tomography (PET)/CT, a huge uneven hypermetabolic mass was seen in the left breast, and there was no evidence of distant organ metastasis except for extensive hypermetabolic LN metastasis (Figure 2b).

Under strong clinical suspicion of inflammatory breast cancer, we performed a core needle biopsy on the tumor itself. Core-cut biopsy revealed a biphasic neoplasm with mild nuclear atypia and low mitotic activity, accompanied by leaf-like processes protruding into dilated ductal spaces, consistent with a low-grade phyllodes tumor. We discussed the discrepancy about the clinical diagnosis and core biopsy results at the preoperative multidisciplinary meeting that included an oncologist, a breast surgeon, a pathologist and a radiologist. We concluded the case was a carcinoma associated with huge phyllodes tumor and decided not to do further preoperative biopsy. The patient underwent a palliative modified radical mastectomy with level II axillary LN dissection. Macroscopic examination of the resection specimen revealed a firm and well-demarcated mass with hemorrhage and necrosis, measuring approximately $24 \mathrm{~cm}$ in the largest dimension (Figure 3a). This mass was composed of two separated tumorous lesions: phyllodes tumor and invasive carcinoma of no special type. Histologically, about $70 \%$ of the tumor area represented a classical

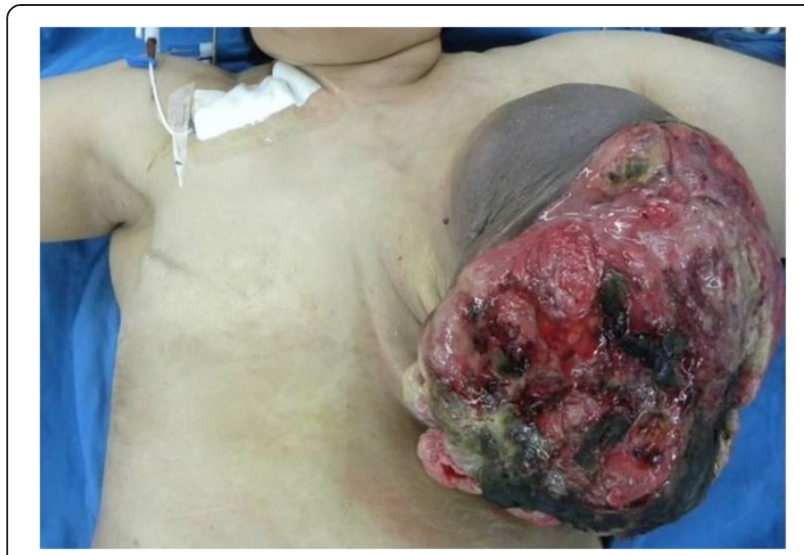

Figure 1 Bulky tumor mass with extensive necrosis and ulceration was seen on left breast. phyllodes tumor showing variable benign to malignant histologic characteristics. The malignant phyllodes tumor was composed of highly cellular stromal cells, moderate and variable nuclear atypia, and increased mitotic activity (>10 mitotic figures per 10 high-power fields) (Figure 3b). In addition, in almost one-third of the tumor, frankly invasive carcinoma of no special type (Figure 3c) with foci of pleomorphic carcinoma was observed (Figure 3d). The skin was thickened and showed pathognomonic dermal lymphatic tumor emboli, consistent with inflammatory carcinoma (Figure 4a). The areas of margin between the two tumor lesions were evaluated by further re-cutting and serial section. On serial section, there was no histologic evidence of transition from phyllodes tumor to invasive ductal carcinoma, so the lesion was thought to be a collision tumor with invasive carcinoma of no special type and malignant PT, rather than ductal carcinoma arising from PT (Figure 4b). Metastatic ductal adenocarcinoma cells were detected in 16 out of 16 dissected axillary lymph nodes. On immunohistochemistry, both epithelial and stromal compartment of the PT as well as the carcinoma cells showed no immunoreactivity for estrogen and progesterone receptors and HER2, except for p53 overexpression on the carcinoma cells.

The wound healed completely without complication. Chemotherapy based on anthracyclines and taxanes was started and radiotherapy was planned. Six months after the operation, and after completion of eight cycle's chemotherapy, the patient developed left supraclavicular and upper chest wall swelling, which was consistent with regional aggravation of metastatic carcinoma and was supported by image findings. She underwent a session of radiation therapy to the chest wall and neck, and platinum-based chemotherapy will be pursued in this patient as further treatment. She is doing well with good compliance to the chemotherapy and radiation therapy.

\section{Discussion}

Although there have been some cases reported of coincidental presentation of breast carcinoma and PT in same breast, most of the cases were the carcinoma that arisesfrom the epithelial component of a malignant or benign PT [5-7]. On review of the literature, we found three cases of true collision tumor with malignant PT and invasive ductal carcinoma in the same breast (Table 1). In case 1 and case 2, collision tumors were composed of malignant PT with sarcomatous differentiation and ductal carcinoma in a separate manner $[8,9]$. But case 3 was a collision tumor of ductal carcinoma within malignant PT without transition. In case 3 , a clonal examination was performed to differentiate the tumor and an analysis of the polymorphic microsatellite markers revealed a divergent loss of heterozygosity $(\mathrm{LOH})$ pattern between stromal and epithelial components of the PT and the carcinoma, thereby 

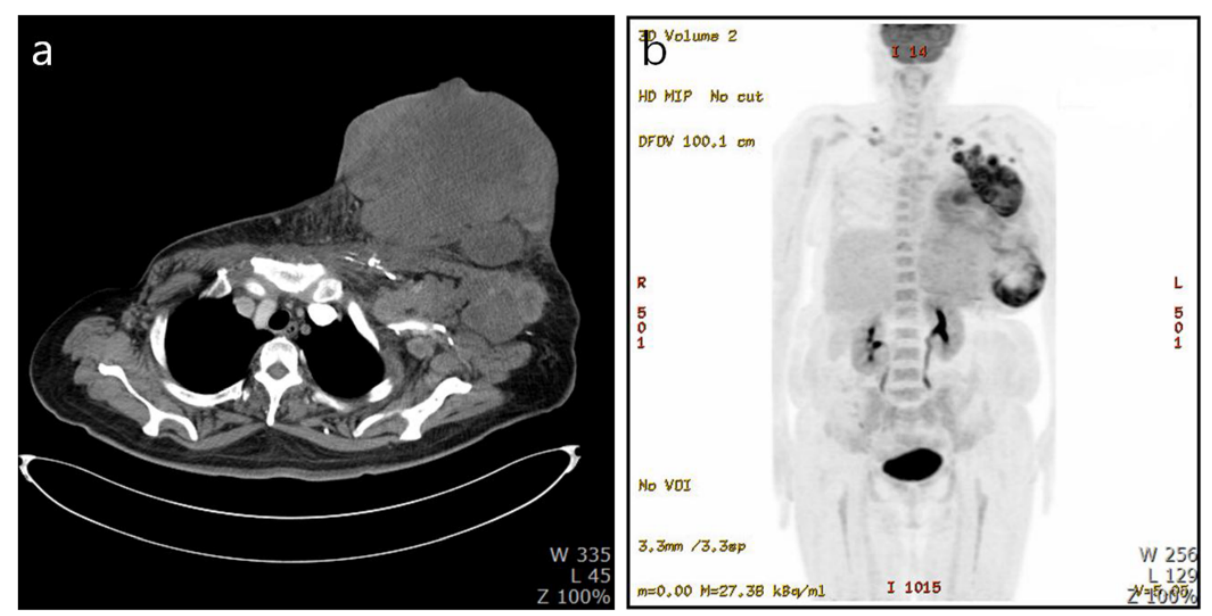

Figure 2 Image findings. (a) Enhanced chest computed tomography (CT) showing a huge breast mass with necrosis and multiple lymph node (LN) enlargements in the left axilla, both supraclavicular area. (b) 18 F-FDG positron emission tomography (PET)/CT revealed a huge uneven hypermetabolic mass and massive hypermetabolic LN metastases without the evidence of distant organ metastasis.

representing a true collision tumor [10]. Similarly, our present case showed distinct presentation of the two separate tumors in same site without any interminglement. In addition, the spindle cell lesion reveals strong expression for vimentin and negative immunoreactivity for Pan-CK,
CK5/6, HM-CK, and the tumor cells in the axillary LNs and skin lesions were compatible with those of invasive ductal carcinoma. So, the lesion was identified as a collision tumor with invasive ductal carcinoma and malignant PT, rather than as a type of carcinosarcoma.
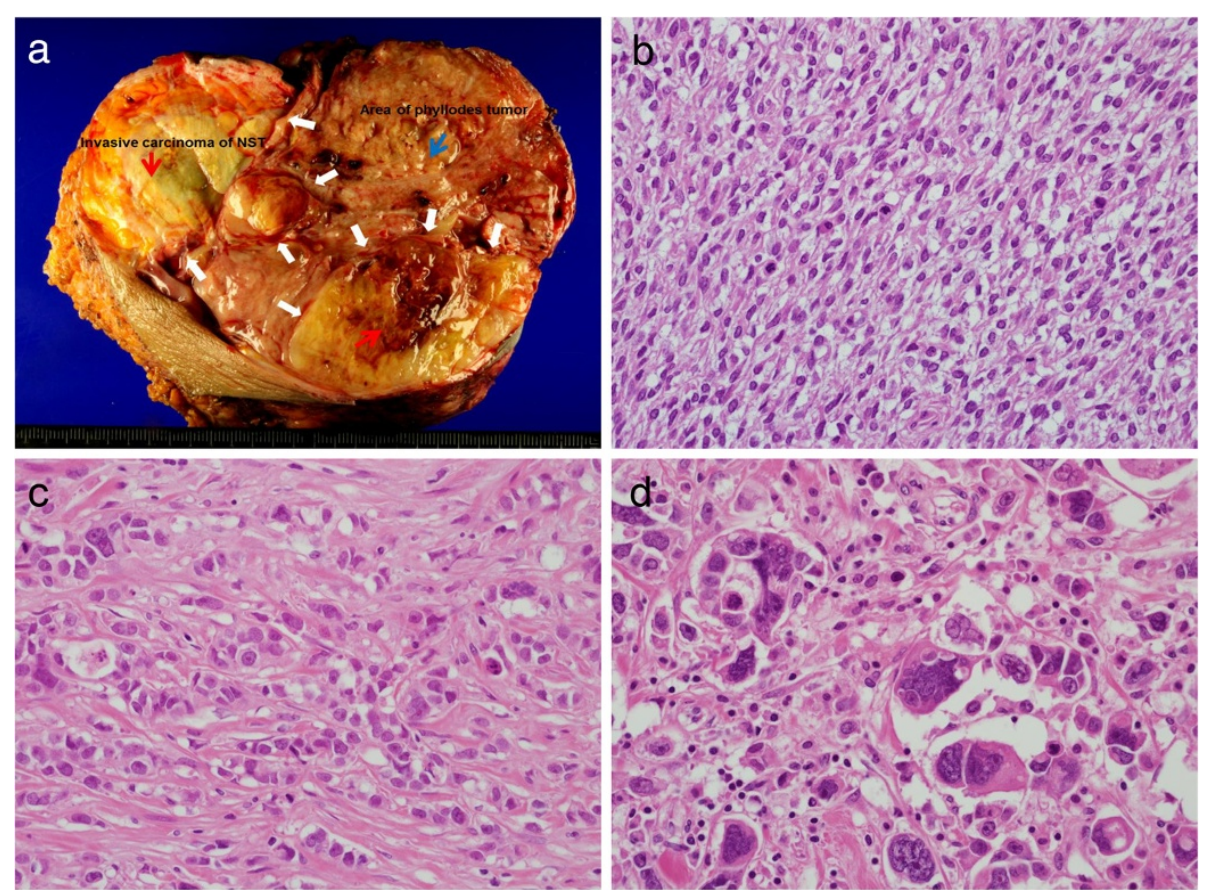

Figure 3 Pathologic findings and photomicrographs of the rumor. (a) Macroscopic examination of the left breast showed a well demarcated mass which composed of two separated tumorous lesions; phyllodes tumor (red arrow) and invasive carcinoma of no special type (blue arrow) with clearly defined margin (white thick arrows). (b) Malignant phyllodes tumor showing a marked stromal cell proliferation with brisk mitotic activity, resembling fibrosarcoma (original magnification $\times 400$ ). (c) Area of invasive carcinoma of no special type (original magnification $\times 400$ ). (d) Area with pleomorphic carcinoma (original magnification $\times 400$ ). 

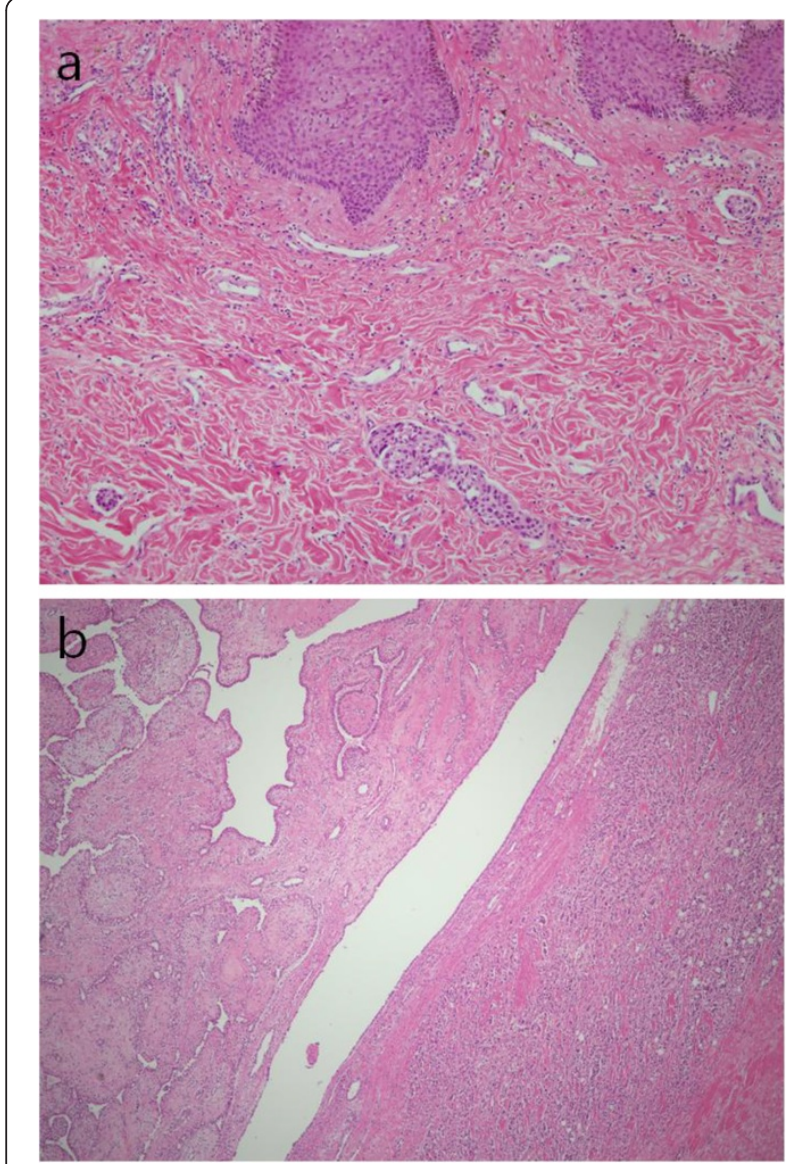

Figure 4 Photomicrographs of the tumor. (a) Thickened skin showing dermal lymphatic tumor emboli (original magnification $\times$ 100). (b) Collision tumor composed of phyllodes tumor and invasive carcinoma of no special type (original magnification $\times 40$ ).

Radiologically, magnetic resonance image (MRI) can be a useful tool for the diagnosis of breast PT. PTs showed internal non-enhanced septations, silt-like patterns in enhanced images and signal changes from T2weighted to enhanced images correlated with the histologic grade [11,12]. MRI showed findings typical of ductal carcinoma, an irregular mass with segmental, regional enhancement demonstrating a rapid increase in signal intensity following contrast enhancement followed by rapid washout. A preoperative MRI could have elevated the diagnostic accuracy in the present case, but we would have been unable to study an MRI because of the huge mass. Also, although there were some differences in metabolism in PET/CT image between PT and ductal carcinoma, they were not pathognomonic. Unfortunately we could not distinguish PT and ductal carcinoma in the preoperative CT and PET/ $\mathrm{CT}$ images because there was a wide area of necrosis in both the huge PT and in the inflammatory breast carcinoma.

The clinical prognosis of collision tumor may be influenced by the subtype and pathologic stage of the more aggressive tumor in the breast. The prognosis for cases consisting of carcinoma and benign PT may be favorable, however, when a carcinoma lesion is detected at an early stage or as an in situ lesion due to a rapidly growing PT [6,7]. In our case, invasive carcinoma of no special type may be considered to have a greater adverse effect on clinical prognosis than the PT due to extensive dermal lymphatic tumor emboli and metastatic ductal carcinoma in the axillary lymph nodes. Furthermore, invasive carcinoma showed triple negative breast cancer (TNBC) with neither expressed hormonal receptors noroverexpressed HER2. Chemotherapy is the only systemic treatment available for TNBC. Cytotoxic therapies, anthracyclines or taxanes, achieved good tumor regression rates in the neo-adjuvant setting, but also showed considerable recurrence. Platinum-containing regimens should be considered as an important treatment option for in both the neoadjuvant and metastatic setting [13]. Even though inflammatory breast carcinoma is often considered a systemic disease, the risk for locoregional recurrence remains an important clinical problem, particularly for patients with triple negative inflammatory breast carcinoma. Therefore, locoregional treatment intensification should be considered on an individual basis. After completion of anthracyclinand taxane-based chemotherapy, our patient showed left upper chest wall and supraclavicular swelling, consistent with regional aggravation of metastatic carcinoma. After the completion of radiation therapy to the chest wall and neck, platinium-based chemotherapy is scheduled.

\section{Conclusions}

We reported a rare case of a collision tumor presented as inflammatory breast carcinoma of a ductal adenocarcinoma and malignant PT. Lacking standardization, treatment of these extremely rare cases should therefore be tailored to treat PT and carcinoma individually. A multimodal approach, including an extensive surgery that includes axilla, may be warranted, as well as an adjuvant treatment with radiation therapy and chemotherapy.

\section{Consent}

Written informed consent was obtained from the patient for publication of this Case report and any accompanying images. A copy of the written consent is available for review by the Editor-in-Chief of this journal. 
Table 1 Summary of reported cases of collision tumor with breast phyllodes tumor and invasive ductal carcinoma

\begin{tabular}{|c|c|c|c|c|c|c|c|c|c|c|c|c|c|c|}
\hline \multirow[t]{2}{*}{ No. } & \multirow[t]{2}{*}{ Author [Reference] } & \multirow[t]{2}{*}{ Year } & \multirow[t]{2}{*}{ Age } & \multicolumn{3}{|c|}{ PT } & \multicolumn{3}{|c|}{ IDC } & \multirow[t]{2}{*}{ Site } & \multirow[t]{2}{*}{ Surgery } & \multirow[t]{2}{*}{ Treatment } & \multirow[t]{2}{*}{ Outcome } & \multirow[t]{2}{*}{ F/U period (mo.) } \\
\hline & & & & Type & Size $(\mathrm{cm})$ & $\overline{\text { LNI }}$ & T stage & Size $(\mathrm{cm})$ & $\mathrm{N}$ stage & & & & & \\
\hline \multirow[t]{2}{*}{1} & L. Auerbach [8] & 2002 & 69 & M & & $(-)$ & $\mathrm{T} 1 \mathrm{~b}$ & & N1 & Separate site & PM AD & ET (TAM) & LR of PT in 40 mo. & 51. \\
\hline & & & & & & & & & & & & & Died of lung metastasis of PT & \\
\hline 2 & Kefeli M et al. [9] & 2008 & 26 & M & 4.5 & $(-)$ & $\mathrm{T} 2$ & 2.5 & $\mathrm{~N} 1(1 / 22)$ & Separate site & MRM & $\mathrm{CT}$ RT & Died in 1 year & 12 \\
\hline 3 & Macher-Goeppinger S et al. [10] & 2010 & 70 & M & 6 & $(-)$ & $\mathrm{T} 2$ & 2.5 & No & Same site & MRM & $\mathrm{CT} R \mathrm{RT}$ & & F/u loss \\
\hline $4^{\mathrm{a}}$ & Shin YD et al. & 2013 & 45 & M & 24 & $(-)$ & T4d & 8 & N3c (16/16) & Same site & MRM & CT RT & Local recurrence of IDC in 6 mo. & 14 \\
\hline
\end{tabular}

"Present case.

PM, partial mastectomy; PT, phyllodes tumor; RT, radiation therapy; TAM, tamoxifen. 


\section{Abbreviations}

CT: Computed tomography; LN: Lymph node; MRI: Magnetic resonance imaging; PET: Positron emission tomography; PT: Phyllodes tumors.

\section{Competing interests}

The authors declare that they have no competing interests.

\section{Authors' contributions}

YJC made substantial contributions to conception and design, and acquisition of data. YDS and SKL drafted the manuscript and revised its final form. KSK performed radiologic interpretation and MJP, HSY, JHK performed pathohistological and immunohistochemistry evaluation. YDS and YJC gave final approval for version to be published. All authors read and approved the final manuscript.

\section{Author details}

'Department of Anesthesiology, Chungbuk National University School of Medicine, Cheongju 361-711, Korea. '2Department of Surgery, Eulji University Hospital, Eulji University School of Medicine, Dunsan-dong 1306, Seo-gu, Daejeon 135-710, Korea. ${ }^{3}$ Department of Radiology, Eulji University Hospital, Eulji University School of Medicine, Dunsan-dong 1306, Seo-gu, Daejeon 135-710, Korea. ${ }^{4}$ Department of Pathology, Eulji University Hospital, Eulji University School of Medicine, Dunsan-dong 1306, Seo-gu, Daejeon 135-710, Korea.

Received: 28 May 2013 Accepted: 25 December 2013

Published: 8 January 2014

\section{References}

1. Miles EF, Jacimore LL: Synchronous bilateral breast carcinoma and axillary non-hodgkin lymphoma: a case report and review of the literature. Case Rep Oncol Med 2012, 2012:685919.

2. Siddiqui FA, Maheshwari V, Alam K, Jain A: Coexistent non-Hodgkins lymphoma and ductal carcinoma breast: diagnosis on fine needle aspiration cytology. Diagn Cytopathol 2011, 39:767-769.

3. Susnik B, Jordi Rowe J, Redlich PN, Chitambar C, Chang CC, Kampalath B: A unique collision tumor in breast: invasive ductal carcinoma and mucosa-associated lymphoid tissue lymphoma. Arch Pathol Lab Med 2004, 128:99-101.

4. Cheung K, Tam W, Chuang E, Osborne MP: Concurrent invasive ductal carcinoma and chronic lymphocytic leukemia manifesting as a collision tumor in breast. Breast J 2007, 13:413-417.

5. Ramdass MJ, Dindyal S: Phyllodes breast tumour showing invasive squamous-cell carcinoma with invasive ductal, clear-cell, secretory, and squamous components. Lancet Oncol 2006, 7:880.

6. Quinlan-Davidson S, Hodgson N, Elavathil L, Shangguo T: Borderline phyllodes tumor with an incidental invasive tubular carcinoma and lobular carcinoma in situ component: a case report. J Breast Cancer 2011, $14: 237-240$

7. Parfitt JR, Armstrong C, O'Malley F, Ross J, Tuck AB: In-situ and invasive carcinoma within a phyllodes tumor associated with lymph node metastases. World J Surg Oncol 2004, 2:46.

8. Auerbach L: Concomitant phyllodes tumour and homo- lateral breast cancer. Lancet Oncol 2002, 3:279.

9. Kefeli M, Yildiz L, Akpolat I, Balci P, Ozen N: The coexistence of invasive ductal carcinoma and malignant phyllodes tumor with liposarcomatous and chondrosarcomatous differentiation in the same breast in a post-osteosarcoma case. Pathol Res Pract 2008, 204:919-923.

10. Macher-Goeppinger S, Marme F, Goeppert B, Penzel R, Schirmacher P, Sinn HP, Aulmann S: Invasive ductal breast cancer within a malignant phyllodes tumor: case report and assessment of clonality. Hum Pathol 2010, 41:293-296

11. Tan H, Zhang S, Liu H, Peng W, Li R, Gu Y, Wang X, Mao J, Shen X: Imaging findings in phyllodes tumors of the breast. Eur J Radiol 2012 81:e62-e69.
12. Alhabshi SM, Rahmat K, Abu Hassan H, Westerhout CJ, Chandran PA: Advanced MRI applications and findings of malignant phyllodes tumour: review of two cases. Jpn J Radiol 2013, 31:342-348.

13. Yagata $H$, Kajiura $Y$, Yamauchi $H$ : Current strategy for triple-negative breast cancer: appropriate combination of surgery, radiation, and chemotherapy. Breast Cancer 2011, 18:165-173.

doi:10.1186/1477-7819-12-5

Cite this article as: Shin et al:: Collision tumor with inflammatory breast carcinoma and malignant phyllodes tumor: a case report and literature review. World Journal of Surgical Oncology 2014 12:5.

\section{Submit your next manuscript to BioMed Central and take full advantage of:}

- Convenient online submission

- Thorough peer review

- No space constraints or color figure charges

- Immediate publication on acceptance

- Inclusion in PubMed, CAS, Scopus and Google Scholar

- Research which is freely available for redistribution

Submit your manuscript at www.biomedcentral.com/submit
C BioMed Central 